\title{
Le paysage éditorial anglo-américain de 1980 à
} 2005

\section{Peter Shillingsburg et Paul Eggert}

Traducteur : Christine Collière

\section{OpenEdition}

Journals

Édition électronique

URL : https://journals.openedition.org/genesis/105

DOI : 10.4000/genesis. 105

ISSN : 2268-1590

\section{Éditeur :}

Presses universitaires de Paris Sorbonne (PUPS), Société internationale de génétique artistique littéraire et scientifique (SIGALES)

\section{Édition imprimée}

Date de publication : 20 juin 2010

Pagination : 35-39

ISBN : 978-2-84050-697-3

ISSN : $1167-5101$

\section{Référence électronique}

Peter Shillingsburg et Paul Eggert, « Le paysage éditorial anglo-américain de 1980 à 2005 », Genesis

[En ligne], 30 | 2010, mis en ligne le 30 mai 2012, consulté le 30 mars 2023. URL : http://

journals.openedition.org/genesis/105; DOI : https://doi.org/10.4000/genesis.105 


\title{
Le paysage éditorial anglo-américain de 1980 à 2005
}

\author{
Peter Shillingsburg et Paul Eggert*
}

$\mathrm{S}$ 'il est vrai que l'édition critique est dans tous les pays une pratique aussi ancienne que celle de la littérature, c'est entre les années trente et cinquante que l'édition de littérature et de théâtre en langue anglaise est devenue une discipline savante ou «scientifique ». D'abord appliquées aux œuvres de la Renaissance, les nouvelles méthodes éditoriales influencèrent bientôt de façon radicale les pratiques d'édition des œuvres littéraires des périodes suivantes. En Amérique du Nord en particulier, cette révolution des pratiques éditoriales se répandit à la fin des années cinquante et prévalut jusqu'à la fin des années soixante-dix. L'inévitable réaction à cette domination, quoique tardive, a rendu les débats éditoriaux anglo-américains des trente dernières années plus riches que dans toute période comparable dans le passé. Parce que les éditeurs britanniques et américains étaient souvent en désaccord sur la théorie, et avaient des pratiques différentes, il est impossible de donner ici une vue d'ensemble de la période 1980-2005 qui soit à la fois brève et complète, d'autant plus qu'une grande partie de l'édition de littérature britannique avait lieu en Amérique et une autre partie dans d'autres pays anglophones, ce qui complique encore notre récit ${ }^{1}$.

Ayant un point de vue interne sur la période récente, nous pensons que le paysage éditorial anglo-américain était et continue à être plus varié qu'il ne peut l'apparaître à des lecteurs européens, même si, bien entendu, nous n'avons pas pu adopter leur point de vue. Ce qui, de l'extérieur, a pu apparaître comme une mutation soudaine des buts que se proposait l'édition anglo-américaine, passant d'un traitement éclectique des intentions finales de l'auteur à l'enregistrement ou à l'archivage des multiples témoins documentaires d'une œuvre, était en réalité une évolution controversée. Parmi les débats qui reviennent le plus souvent, on trouve en première place ceux qui portent sur les intentions des agents de modifications textuelles, le choix du manuscrit ou texte de base (copy-text), les principes d'émendation, les éditions juxtaposant plusieurs versions d'un même texte, et des réflexions de fond sur ce que recouvrent les termes « texte », « document » et « œuvre 2 ».

* Cet article représente une version raccourcie de l'introduction au numéro 6 de la revue Ecdotica (2009), consacré à la critique textuelle anglo-américaine. Nous remercions la rédaction d'Ecdotica de nous avoir autorisés à le traduire.

1. Le meilleur ouvrage sur la théorie et la pratique éditoriales du Xxe siècle en Grande-Bretagne et en Amérique est celui de David Greetham (dir.), Scholarly Editing: A Guide to Research (New York, Modern Language Association, 1996) dont une traduction italienne vient d'être annoncée par les Presses universitaires de Bologne. Ce guide retrace les évolutions des pratiques éditoriales de l'édition biblique et classique, de l'édition de textes médiévaux et de textes en anglais moderne naissant, de la Renaissance (en particulier de l'édition shakespearienne), ainsi que de l'édition des textes des XVIII ${ }^{\mathrm{e}}$, XIX ${ }^{\mathrm{e}}$ et $\mathrm{XX}^{\mathrm{e}}$ siècles. Il contient également des articles sur l'édition critique allemande, française, italienne et espagnole. On trouve aussi une étude approfondie et une évaluation des débats éditoriaux dans l'ouvrage de George T. Tanselle, Textual Criticism Since Greg: A Chronicle, 1950-2000 (Charlottesville, Bibliographical Society, 2005). Tanselle a également rassemblé plusieurs bibliographies détaillées jusqu'à 2002 sur <www.rarebookschool.org/tanselle>, consulté le 25 octobre 2009. Parmi d'autres résumés des pratiques éditoriales angloaméricaines, citons Ronald Gottesman, Art and Error: Modern Textual Editing (Bloomington, Indiana University Press, 1970) ; Geert Lernout, «La critique textuelle anglo-américaine : une étude de cas », Genesis, n 9 (1996), p. 45-65 ; Peter Shillingsburg, « Anglo-amerikanische Editionwissenschaft: Ein knapper Überblick », dans R. Nutt-Kofoth, B. Plachta, H. T. M. van Vliet et H. Zwerschina (dir.), Text und Edition: Positionen und Perspektiven (Berlin, Erich Schmidt, 2000), p. 143-164 ; il existe une version revue et corrigée, en anglais, de cet article : «On Being Textually Aware », Studies in American Naturalism, 1 (2006), p. 170-195 ; Ernst Honigmann, « The New Bibliography and its Critics », dans L. Erne et M.J. Kidnie (dir.), Textual Performances : The Modern Reproduction of Shakespeare's Drama (Cambridge, Cambridge University Press, 2004), p. 77-93 ; et Paul Eggert, Securing the Past: Conservation in Art, Architecture and Literature (Cambridge, Cambridge University Press, 2009), chap. VIII et IX.

2. Pour une vue d'ensemble et une discussion, voir P. Eggert, Securing the Past, op. cit., chap. VII-X. 
À partir des années soixante-dix, une augmentation considérable du nombre et de la variété des éditions d'œuvres postérieures à la Renaissance est venue progressivement alimenter ces discussions. Contrairement au corpus shakespearien, dont les manuscrits originaux sont presque intégralement perdus (et dont l'édition repose donc essentiellement sur l'analyse bibliographique de livres imprimés), les siècles suivants ont livré des archives littéraires dont la richesse textuelle et la variété ne se pliaient pas aisément aux principes éditoriaux traditionnels dits «du témoin unique » ou « du meilleur témoin ». Une transformation de la pratique éditoriale anglo-américaine était inévitable, mais fut ralentie par les freins institutionnels des organismes de financement et de contrôle, peu ouverts à l'innovation, par les attentes des maisons d'édition ainsi que par l'effet de retardement des éditions d'œuvres complètes encore en cours dont les principes éditoriaux avaient été décidés dans les années soixante et soixante-dix.

$\mathrm{Au}$ début $\mathrm{du} \mathrm{xx}^{\mathrm{e}}$ siècle, la théorie et la pratique éditoriales anglo-américaines s'étaient détachées des traditions éditoriales habituellement (mais à tort) associées à Lachmann, qui se fondent sur l'importance accordée à la stemmatique $^{3}$. Les travaux d'A.E. Housman sur des textes classiques en latin, notamment son édition des œuvres de Manilius, ont marqué ce changement. S'opposant aux pratiques éditoriales arbitrairement liées aux « meilleurs documents », Housman exerça son intelligence critique sur tous les aspects de l'œuvre à éditer. Ceci impliquait de retrouver les versions conservées dans d'anciens manuscrits mais en exerçant ses facultés critiques afin de juger de la fiabilité de leurs témoins textuels aux points de variations, plutôt qu'en faisant appel de façon générale à leur autorité documentaire ${ }^{4}$. R.B. McKerrow, son contemporain, dont la réputation d'éditeur était aussi grande, suivit Housman dans l'exercice des facultés critiques pour le choix du texte le plus digne de foi, mais recommanda de conserver toutes les leçons de la source finalement adoptée comme texte de base, sauf dans le cas d'erreurs démontrables. Ces deux éditeurs rejetaient, par des moyens différents, une adhérence trop naïve aux documents sources et une utilisation trop libre des émendations spéculatives. Ce qui poussa McKerrow à refuser la liberté que se permettait Housman dans l'exercice de son jugement critique, c'est qu'il n'avait pas confiance dans le discernement de bien des éditeurs qui l'avaient précédé et qu'il ne se dissimulait pas que des zones d'ombre demeuraient autour des agents responsables des modifications dans les textes pour lesquels n'avaient survécu que peu, ou pas, de documents originaux.

La recherche d'un juste équilibre entre ces deux tendances sous-tend presque toutes les discussions et tous les développements de la théorie et de la pratique éditoriales anglo-américaines du $\mathrm{xx}^{\mathrm{e}}$ siècle. Au milieu du siècle, la balance penche du côté d'Housman, lorsque W.W. Greg rejette la prudence de McKerrow, affirmant qu'elle subit la «tyrannie du texte de base 5 ». Elle force les éditeurs à accepter des leçons malencontreuses parce qu'elles se trouvent être dans le document le plus digne de foi, mais n'atteignent pas le statut d'erreur démontrable qui aurait permis de les rejeter. Au contraire, Greg préconise de distinguer les éléments " substantifs » des éléments « accidentels » car, selon les pratiques habituelles, les aspects substantifs du texte (c'est-à-dire les mots et l'ordre des mots) sont plus souvent sous le contrôle des auteurs, tandis que les aspects formels d'un document (tels que les majuscules, l'orthographe, la division des mots et la ponctuation) sont davantage susceptibles d'être sous le contrôle des compositeurs.

3. Voir G. Fiesoli, La genesi del lachmannismo (Firenze, Sismel Edizioni del Galluzzo, 2000), dont un compte rendu a été fait par L. Castaldi dans Ecdotica, $\mathrm{n}^{\circ}$ 1, p. 55-65; en anglais, P. L. Schmidt, « Lachmann's Method. On the History of a Misunderstanding », dans The Uses of Greek and Latin, dir. A.C. Dionisotti (London, Warburg Institute, 1988), p. 227-236.

4. A.E. Housman, «The Application of Thought to Textual Criticism », Proceedings of the Classical Association, 18 (1922), p. 67-84 ; reproduit dans John Carter (dir.), Selected Prose (Cambridge, Cambridge University Press, 1961), p. 131-150. Voir aussi la discussion des idées d'Housman par Ernst Honigmann dans « The New Bibliography and its Critics », dans Erne and Kidnie (dir.), Textual Performances, op. cit., p. 77-93. Voir D.C. Greetham, «Textual and Literary Theory: Redrawing the Matrix », publié originellement dans Studies in Bibliography, $n^{\circ} 42$ (1989), p. 1-24; et Jeremy Lawrance, «Stoppard, Housman and the mission of textual criticism », dans Ecdotica, n ${ }^{\circ}$, p. 187-205.

5. W.W. Greg, «The Rationale of Copy-Text », Studies in Bibliography, $\mathrm{n}^{\circ} 3$ (1951), p. 19-36; traduit en italien dans Pasquale Stoppelli, Filologia dei testi a stampa, Nuova edizione aggiornata (Cagliari, CUEC/Centro di Studi Filologici Sardi, 2008), p. 39-58. 
Pour échapper à la tyrannie du texte de base, Greg recommande de choisir comme texte de base le document dans lequel les aspects accidentels représentent le plus probablement le travail de l'auteur et d'incorporer à cette version les variantes substantives présentes dans d'autres documents qui portent la preuve de l'intervention de l'auteur. Un éditeur, selon lui, est généralement mieux placé pour opérer une discrimination entre des variantes substantives qu'il n'est possible de le faire entre différentes formes accidentelles. Le résultat serait une édition éclectique dont le texte serait tiré de deux ou plusieurs sources. Il est important de noter que Greg n'invoquait la règle du texte de base que lorsque les preuves des préférences de l'auteur en matière de forme ne pouvaient être établies avec précision. Sa prétendue théorie de l'édition du texte de base (comme certains commentateurs devaient s'y référer par la suite) ne fut jamais pour lui qu'une règle générale.

Avec le soutien de Fredson Bowers puis, plus tard, de George Thomas Tanselle, ainsi que celui des organismes de financement gouvernementaux, les positions de Greg s'imposèrent dans des projets éditoriaux majeurs de la Renaissance au XIX ${ }^{\mathrm{e}}$ siècle et même au $\mathrm{XX}^{\mathrm{e}}$ siècle, en particulier pour des œuvres d'auteurs américains. En 1975, le Centre pour l'édition d'auteurs américains (Center for Editions of American Authors) avait supervisé et approuvé cent quarante volumes d'éditions critiques qui tous se conformaient à la prétendue " école Greg-Bowers ». La fin des années soixante-dix voit le triomphe de l'approche Housman-Greg-Bowers de l'édition critique et des textes éclectiques établis selon des principes raisonnés.

En 1976, le Fonds national pour les sciences humaines (National Endowment for the Humanities) décida de financer directement les éditeurs scientifiques, sans passer par le CEAA, et de transférer les fonctions d'évaluation au Centre pour les éditions critiques de l'Association pour les langues modernes (CSE). Ceci eut pour résultat un élargissement du domaine du CSE qui s'étendit aux littératures britanniques et non anglophones, ainsi qu'une approche plus inclusive des pratiques éditoriales.

En 1980, au début de la période qui nous concerne, l'expérience grandissante des éditeurs de textes modernes, pour lesquels existent de multiples documents auctoriaux, commençait à transformer le domaine éditorial. Au lieu de se consacrer à la quête d'un texte unique, authentiquement auctorial, les éditeurs prenaient progressivement conscience d'un surplus textuel, qui rendait possible l'établissement de textes multiples à des fins multiples. Ainsi Hershel Parker mit au jour de nombreux documents prouvant que les intentions «finales » d'un auteur allaient fréquemment à l'encontre de ses intentions « initiales » et que lorsque des auteurs âgés révisaient les textes de leur jeunesse, cela pouvait avoir des résultats regrettables d'un point de vue critique 6 . De même, Don Reiman commença à préconiser les éditions multiples («versioning ») comme étant une approche éditoriale plus riche que celle de l'édition éclectique, qui avait tendance à homogénéiser les textes. Cette tendance consistant à considérer la présentation de textes auctoriaux multiples comme un but légitime de l'édition critique atteignit son point culminant en 1985 , à New York, lors de la conférence de l'Association pour la recherche textuelle (Society for Textual Scholarship ou STS) : les arguments de Jerome McGann, préconisant une manière complètement différente d'envisager la question de l'autorité textuelle, y trouvèrent un écho culturel et commencèrent à exercer leur pouvoir de séduction.

Avec une communication en 1982 sur la critique textuelle et l'interprétation littéraire (publiée en 19857), et un livre qui fit grand bruit en 1983 dans le monde relativement placide de l'édition d'auteurs, A Critique of Modern Textual Criticism ${ }^{8}$, McGann entreprit ce qui revient à une réhabilitation de la résistance de McKerrow à l'éclectisme et à l'exercice du jugement éditorial individuel. Cependant, le fond de son argument ne repose pas sur une méfiance vis-à-vis du jugement éditorial, mais sur le fait que les textes littéraires publiés portent la trace historique de l'influence collective de la communauté sociale, économique et discursive sur le texte publié originellement. L'histoire de ce processus social serait altérée si les éditeurs tournaient le dos aux textes effectivement lus par les générations précédentes. En adaptant les idées

6. H. Parker, Flawed Texts and Verbal Icons, Evanston, Northwestern University Press, 1984.

7. «The Monks and the Giants: Textual and Bibliographical Studies and the Interpretation of Literary Works », dans Jerome McGann (dir.), Textual Criticism and Literary Interpretation (Chicago, University of Chicago Press, 1985), p. 180-199.

8. A Critique of Modern Textual Criticism (Chicago, University of Chicago Press, 1983). 
de D.F. McKenzie sur le livre comme forme expressive dans Bibliography and the Sociology of Texts, l'argument de McGann poussait bien plus loin les travaux de James Thorpe des années soixante-dix et sera à son tour développé par ceux de G.E. Bentley Jr et de Jack Stillinger dans les années quatre-vingt-dix (sur William Blake et John Keats, respectivement) et par les travaux de George Bornstein dans les années quatre-vingt-dix et deux mille (sur W.B. Yeats) ${ }^{9}$.

Parmi d'autres critiques, c'est Bornstein qui nous a permis de voir la signification inhérente aux différentes dispositions des poèmes des recueils de W.B. Yeats, à leur présentation sur la page, et même à leur reliure. En révélant, dans son étude novatrice de 1988, les diverses manières dont Blake, en illustrant et en imprimant luimême ses poèmes, a démonté les présupposés éditoriaux à propos du support imprimé, Bentley a ouvert la voie pour une appréciation plus complète de la signification physique ou matérielle de la page imprimée.

La capacité actuelle des recherches bibliographiques à remettre en question ses propres présupposés fondamentaux sera également illustrée, au milieu des années quatre-vingt, par les travaux de Harold Love sur la survivance de la diffusion manuscrite plusieurs siècles après l'invention de l'imprimerie.

Un autre déplacement d'intérêt majeur avait lieu à peu près au même moment. Au lieu d'être considérés comme un «produit » final - qu'il s'agisse du produit des intentions de l'auteur ou des agencements du complexe social de production et de distribution - les textes commencent à être décrits comme des processus pouvant être compris grâce à l'étude des manuscrits et, si jamais ils étaient édités, devant être présentés de telle façon que la progression de l'œuvre, de sa composition à sa production, en passant par les révisions, puisse être retracée. Dans les cercles éditoriaux anglo-américains, ce courant n'a été appelé « critique génétique » qu'à partir du moment où l'influence de la critique génétique française a commencé à infléchir les pratiques anglo-américaines, dans les années quatre-vingt-dix ${ }^{10}$; mais dès les années quatre-vingt, les éditeurs et les critiques se sont de plus en plus intéressés au processus d'évolution du texte. Ils ont de mieux en mieux compris que l'instabilité était une inéluctable modalité de la condition textuelle.

Au cours des années qui suivirent, la théorie éditoriale s'étendit rapidement pour absorber les nouvelles avancées concernant le phénomène de l'instabilité textuelle. Joseph Grigely devait décrire très justement la situation en 1991, avec tout ce qu'elle impliquait :

[...] un moment d'écriture par l'auteur, le moment de la publication, ou le moment de la lecture - ou n'importe quel moment entre deux. Un [...] moment de stase [...] une série de moments d'inscription, certains auctoriaux, d'autres non, certains autorisés, d'autres non ; des moments de stase [...] qui sont mieux définis par ce qu'ils taisent que par ce qu'ils disent : ils nous laissent face à un texte désincarné, décontextualisé, qui ne signifie rien s'il n'est pas lié à un agent de sens - à un interprète 11 .

Les multiples instances textuelles, produites par les divers agents de modification textuelle au cours de ce processus, sont analysées par Peter Shillingsburg comme des « actes d'écriture12 ». Ce terme, qui prend place dans une théorie du texte plus vaste, a été inventé en 1997, mais se fondait sur un article paru dans Studies in Bibliography en 1991.

9. D.F. McKenzie, Bibliography and the Sociology of Texts, Panizzi Lectures, 1985 (London, British Library, 1986) ; J. Thorpe, Principles of Textual Criticism (San Marino, Huntington Library, 1972) ; J. Stillinger, Multiple Authorship and the Myth of Solitary Genius (New York, Oxford University Press, 1991) ; et G. Bornstein, « How to Read a Page: Modernism and Material Textuality », Studies in the Literary Imagination, 32.1, 1999, p. 30-57 et Material Modernism: The Poetics of the Page (Cambridge, Cambridge University Press, 2001).

10. Daniel Ferrer et Hans Walter Gabler en ont présenté des exemples lors des conférences de la STS, et à la conférence « Iconic Page », commémorée dans George Bornstein et Theresa Tinkle (dir.), The Iconic Page (Ann Arbor, University of Michigan Press, 1998). Voir aussi Jed Deppman, Daniel Ferrer et Michael Groden (dir.), Genetic Criticism: Texts and Avant-Textes (Philadelphia, University of Pennsylvania Press, 2004); et Dirk Van Hulle, Manuscript Genetics, Joyce's Know-How, Beckett's Nohow (Tallahassee, University Press of Florida, 2009).

11. Joseph Grigely, «The Textual Event», dans Philip Cohen (dir.), Devils and Angels: Textual Editing and Literary Theory (Charlottesville, University of Virginia Press, 1991), p. 167-194 [p. 172].

12. Un acte scriptural, comme l'énonciation pour les actes de parole, est entrepris à un moment et en un lieu spécifique par les auteurs, les artisans de la production et les lecteurs. Le sens, pour chaque acte scriptural, dépend non seulement de l'agent de modification textuelle et des circonstances qui ont provoqué la modification, mais aussi de l'agent et des circonstances de réception. Voir P. Shillingsburg, From Gutenberg to Google: Electronic Representations of Literary Texts (Cambridge, Cambridge University Press, 2006). 
Les travaux de Marta Werner sur Emily Dickinson, parus dans les années quatre-vingt-dix, et ceux de John Bryant sur Melville, publiés dans les années deux mille, devaient proposer des exemples radicaux et extrêmement développés de la version anglo-saxonne de la théorie, des commentaires et de la pratique du texte en tant que processus $^{13}$. Une des implications de l'instabilité textuelle récemment découverte était la nécessité de refondre les conceptions établies des relations entre les dimensions documentaires et textuelles de l'œuvre : un exemple en est la réflexion de Paul Eggert sur ce sujet parue en 1994 dans TEXT14. L'essor de l'Association pour la recherche textuelle et de sa revue TEXT témoigne également du bouillonnement intellectuel du milieu éditorial dans la période en question ${ }^{15}$.

À la fin des années quatre-vingt, les nouvelles doctrines de l'instabilité textuelle et du processus textuel ont rencontré, par une coïncidence fortuite, l'invention de l'hypertexte, puis, vers 1991-1992, Internet, auquel on avait désormais accès sur les ordinateurs personnels. Un nouvel avenir s'ouvrait pour l'édition critique. Tout à coup, les limitations matérielles du codex semblaient ne plus avoir lieu d'être, potentiellement du moins, et donc ne plus avoir systématiquement le dernier mot lors des discussions sur les possibilités d'édition. Les premiers espoirs frustrés, puis, ces dernières années, le développement plus progressif et réfléchi de ce support pour les besoins des éditions savantes, demanderaient à être racontés, mais la complexité technique et théorique d'une telle histoire, encore en cours, dépasse cependant le cadre du présent tableau ${ }^{16}$.

Traduit de l'anglais par Christine Collière

13. Voir M. Werner, Emily Dickinson's Open Folios: Scenes of Reading, Surfaces of Writing (Ann Arbor, University of Michigan Press, 1995) et Martha Nell Smith, Rowing in Eden: Rereading Emily Dickinson (Austin, University of Texas Press, 1992) ; J. Bryant, The Fluid Text: A Theory of Revision and Editing for Book and Screen (Ann Arbor, University of Michigan Press, 2002) et Melville Unfolding (Ann Arbor, University of Michigan Press, 2008).

14. «Document and Text: The "Life" of the Literary Work and the Capacities of Editing », TEXT, n ${ }^{\circ}$ 7, 1994, p. 1-24.

15. L'Association pour la recherche textuelle a été fondée en 1979 et a publié TEXT chaque année de 1984 à 2007, lorsque le titre du journal et ses statuts ont été modifiés pour devenir Textual Cultures.

16. Pour une vue d'ensemble de ces questions, voir l'avant-propos de G. Thomas Tanselle dans L. Burnard, K. O'Brien O'Keeffe, J. Unsworth (dir.), Electronic Textual Editing (New York, Modern Language Association of America, 2006). Voir aussi P. Eggert, «These post-philological days... », dans Ecdotica, ${ }^{\circ}$ 2, p. 80-98, et D.C. Greetham, «Philology Redux? », dans Ecdotica, n 3, p. 103-128.

Paul Eggert travaille dans le domaine de la théorie éditoriale et des philosophies de la conservation et de la restauration. Son ouvrage Securing the Past: Conservation in Art, Architecture and Literature est paru chez Cambridge University Press en 2009. Il a préparé des éditions savantes d'œuvres de D.H. Lawrence, Henry Kingsley et Rolf Boldrewood, et il a été l'éditeur principal des Academy Editions of Australian Literature. Il est directeur de recherche à l'Australian Research Council, en poste à l'ADFA de l'université de New South Wales à Canberra.

PaulEggert, p.eggert@adfa.edu.au

Peter Shillingsburg est titulaire de la Svaglic Chair of Textual Studies à l'université Loyola de Chicago. Il est l'éditeur principal des œuvres de William Makepeace Thackeray et est l'auteur de nombreux ouvrages sur Thackeray et sur les études textuelles, dont le plus récent est From Gutenberg to Google: Electronic Representations of Literary Texts (Cambridge University Press, 2006).

Peter Shillingsburg, pshillingsburg@luc.edu 